\title{
Prognostic value of glypican-1 for patients with advanced pancreatic cancer following regional intra-arterial chemotherapy
}

\author{
JING-YU QIAN $^{1,2}$, YU-LIN TAN ${ }^{2}$, YANG ZHANG $^{2}$, YONG-FEI YANG $^{3}$ and XIAO-QIANG LI ${ }^{1}$ \\ ${ }^{1}$ Department of Vascular Surgery, The Second Affiliated Hospital, Soochow University, Suzhou, Jiangsu 215004; \\ ${ }^{2}$ Department of Interventional Radiology, The First Affiliated Hospital of Bengbu Medical College, Bengbu, \\ Anhui 233004; ${ }^{3}$ School of Life Science, Beijing Institute of Technology, Beijing 100081, P.R. China
}

Received May 23, 2017; Accepted February 1, 2018

DOI: $10.3892 / \mathrm{ol} .2018 .8701$

\begin{abstract}
Regional intra-arterial chemotherapy (RIAC) is a potential alternative treatment for advanced pancreatic cancer (APC) with fewer adverse effects than other treatment options. However, specific biomarkers to determine the prognosis of patients with APC have thus far, been unsatisfactory. Glypican-1 (GPC1) in exosomes has been identified as an early diagnostic biomarker for pancreatic adenocarcinoma. The aim of the present study was to investigate whether the presence of GPC1 in extracellular vesicles (EVs) could serve as a predictor of RIAC outcome for patients with APC. EVs in circulation were isolated and the percentage of $\mathrm{GPC}^{+} \mathrm{EVs}$ was measured using flow cytometry. Compared with healthy individuals, the levels of $\mathrm{GPC1}^{+} \mathrm{EVs}$ were significantly increased in patients with APC $(\mathrm{P}<0.01)$. Following RIAC treatment, the percentage of $\mathrm{GPC}^{+} \mathrm{EVs}$ was decreased $(\mathrm{P}=0.023)$. Furthermore, patients with APC exhibiting a greater decrease of $\mathrm{GPC1}^{+} \mathrm{EVs}$ experienced improved overall survival (OS) rates. In summary, the present study provides insights into identifying GPC1 as a novel prognostic biomarker for patients with APC following RIAC treatment.
\end{abstract}

\section{Introduction}

Pancreatic cancer has a poor prognosis, with a 5-year survival rate of $<7 \%$ due to early local invasion and distant metastasis $(1,2)$. At the time of first diagnosis, $85 \%$ of patients will have progressed to an advanced stage and are therefore not eligible to undergo curative resection $(3,4)$. For advanced pancreatic cancer (APC), regional intra-arterial chemotherapy (RIAC), which delivers drugs into the carcinoma tissue more selectively, is considered to be a potential alternative treatment

Correspondence to: Dr Xiao-Qiang Li, Department of Vascular Surgery, The Second Affiliated Hospital, Soochow University, 1055 Sanxiang Road, Suzhou, Jiangsu 215004, P.R. China E-mail: flytsg@126.com

Key words: glypican-1, pancreatic adenocarcinoma, regional intra-arterial chemotherapy, extracellular vesicles for APC with less adverse systemic effects (5-8). Thus far, cancer antigen 19-9 (CA19-9) is routinely used to predict the outcome of patients with APC following surgery or chemotherapy. However, there are limitations for the use of CA19-9 as a biomarker, which include its high expression in other diseases such as pancreatitis and obstructive jaundice (9). Therefore, it is necessary to identify reliable biomarkers with high sensitivity and specificity to determine the prognosis of patients with pancreatic adenocarcinoma following interventional therapy.

Extracellular vesicles (EVs), including exosomes, are derived from endosomes and contain a subset of proteins, lipids and nucleic acids (10). EVs may be secreted by cancer cells and transferred into the circulation (11). As a type of EV, exosomes represent a novel diagnostic biomarker for multiple types of cancer (12). Glypican-1 (GPC1) is a member of the heparin sulfate proteoglycans family (13). GPC1 functions as a co-receptor for heparin-binding growth factors and serves notable functions in multiple signaling pathways, including the Wnt and mitogen-activated protein kinase pathways (14). GPC1 is highly expressed in pancreatic cancer tissue and promotes tumor invasion and metastasis $(15,16)$. Downregulation of GPC1 decreases the tumorigenicity of pancreatic cancer cells, whereas high levels of GPC1 are associated with poor survival in patients with pancreatic cancer (17). Melo et al (13) identified that $\mathrm{GPC}^{+}$circulating exosomes could be used as a tool for detecting and monitoring early pancreatic cancer. Furthermore, the study also revealed that $\mathrm{GPCl}^{+}$circulating exosomes were associated with improved survival of patients with APC.

Clinical trials demonstrated that RIAC improved the resectability rates for $\mathrm{APC}$ and was well tolerated by patients $(18,19)$. Although $\mathrm{GPCl}^{+}$circulating exosomes were positively associated with the burden of pancreatic cancer and prognosis following surgical treatment (13), whether $\mathrm{GPCl}^{+} \mathrm{EVs}$ could be used to predict the outcome of patients with APC following RIAC treatment remained unresolved.

In the present study, a spin column was used to isolate EVs from the plasma of healthy individuals and patients with APC pre- and post-RIAC treatment. The levels of $\mathrm{GPCl}^{+} \mathrm{EVs}$ were measured with flow cytometry. Compared with healthy controls, it was identified that the levels of $\mathrm{GPC1}^{+} \mathrm{EVs}$ were 
significantly increased in patients with APC. Furthermore, $\mathrm{GPC}^{+} \mathrm{EVs}$ levels were reduced following RIAC therapy. It was also revealed that survival rates were significantly increased in patients with a greater decrease in $\mathrm{GPC}^{+} \mathrm{EVs}$. In summary, the present study provides an insight that $\mathrm{GPC1}^{+} \mathrm{EVs}$ may be useful as a novel predictor to evaluate the outcome of RIAC for patients with APC.

\section{Materials and methods}

Patient sample collection. A total of 28 patients were recruited between January 2013 and October 2016 at The First Affiliated Hospital of Bengbu Medical College (Bengbu, China). All patients were required to meet the following criteria: i) APC was confirmed by pathology or imaging (including computed tomography or magnetic resonance imaging); ii) patients were considered not to be suitable for surgical resection; iii) patients received RIAC treatment at The First Affiliated Hospital of Bengbu Medical College; iv) patients did not undergo any treatment besides RIAC therapy. For healthy controls, 16 age- and gender-matched individuals were enrolled under strict criteria, excluding any patients with acute, chronic or other malignant diseases, including chronic disorders involving vital organs (heart, lung, kidney and brain), serious inflammatory diseases (pancreatitis, diabetes and rheumatoid arthritis), cancers (prostate, ovary and breast cancer), chronic use of drugs may induce pancreas-associated diseases (didanosine and azathioprine) and malnutrition conditions (chronic diarrhea).

Plasma was isolated from $10 \mathrm{ml}$ peripheral blood (as described below) and stored at $-80^{\circ} \mathrm{C}$ until analysis. For 28 patients with APC, peripheral blood was drawn prior to RIAC and on the 10th day following RIAC treatment. Written informed consent was obtained from all study participants prior to participating in the study. The study was approved by the Institutional Research Ethic Board at The Bengbu Medical College.

Clinical procedure. Written informed consent for the RIAC procedure was obtained from all patients or their relatives. Gemcitabine (GEM) is a routinely used drug for treating patients with pancreatic cancer in China. Right femoral artery puncture and intubation was performed using the Seldinger method with a 5-Fr arterial sheath (20). Next, a celiac artery and superior mesenteric artery angiography was performed. The potentially infused artery was evaluated carefully prior to the clinical procedure. GEM was administered at a dose of between 1,000 and $1,200 \mathrm{mg} / \mathrm{m}^{2}$ followed by cisplatin at $90 \mathrm{mg} / \mathrm{m}^{2}$ and/or 1,000 mg tegafur. Among them, 23 patients treated with cisplatin $(n=15)$ or tegafur $(n=8)$ alone, and 5 patients were treated with the combination of cisplatin and tegafur. For patients with lesions in the head of the pancreas, the drugs were primarily infused via the gastroduodenal artery. For patients with lesions in the pancreatic body or tail, the drugs were mainly infused via the splenic artery. All the clinical procedures were performed under local anesthesia. Necessary care including antiemetics, liver protection and anti-inflammatory treatment was administered following RIAC treatment.

Clinicopathological data collection. Clinicopathological information, consisting of age, gender, tumor location (head, body and tail) and metastases condition, were collected from electronic medical records. Tumor-Node-Metastasis classification was assessed based on the images captured, including computed tomography images (21). CA19-9 and carcinoembryonic antigen (CEA) levels were examined in the clinical laboratory. Survival time was defined from the first diagnosis of pancreatic cancer to mortality or the final follow-up date.

EV isolation. Prior to purification of EVs, plasma was thawed on ice. EVs were isolated utilizing a spin column-based method (77044; exoRNeasy Serum/Plasma Maxi Kit; Qiagen $\mathrm{GmbH}$, Hilden, Germany) according to the manufacturer's protocol (22). The binding buffer, wash buffer and elution buffer are supplied with the kit. Briefly, $500 \mu 1$ plasma was filtered through a $0.22-\mu \mathrm{m}$ filter to exclude cell debris. Filtered plasma was then mixed with binding buffer and added to the membrane affinity column for to bind EVs. Following centrifugation, the flow-through was discarded and wash buffer was added to the column to remove non-specific substances. Finally, elution buffer was added to the spin column membrane and EVs were collected through centrifugation at 5,000 $\mathrm{x}$ g for $5 \mathrm{~min}$ at room temperature.

Transmission electron microscopy. The isolated EVs were fixed in $2 \%$ paraformaldehyde at $4^{\circ} \mathrm{C}$ for $20 \mathrm{~min}$. A formvar-coated copper grid was floated on the fixed sample for $20 \mathrm{~min}$. Then samples were fixed in $1 \%$ glutaraldehyde at $4^{\circ} \mathrm{C}$ for 5 min prior to staining with $2 \%(\mathrm{w} / \mathrm{v})$ uranyl oxalate for $5 \mathrm{~min}$. Finally, the sections were embedded in $0.4 \%$ (w/v) uranyl acetate and $1.8 \%(\mathrm{w} / \mathrm{v})$ methyl cellulose on ice for $10 \mathrm{~min}$. Excess liquid was removed from the grid using filter paper and the grid was allowed to dry for several minutes. Images were captured using a transmission electron microscope (Tecnai G2 spirit BioTwin, FEI, 120 kV; Fei Company; Thermo Fisher Scientific, Inc., Waltham, MA, USA).

Flow cytometry analysis. The isolated EVs were incubated with $4 \mu \mathrm{m}$ aldehyde/sulfate latex beads (Invitrogen; Thermo Fisher Scientific, Inc.) at room temperature for $15 \mathrm{~min}$. This mixture was dissolved in $1 \mathrm{ml}$ PBS at room temperature for $1 \mathrm{~h}$ and the reaction was quenched with $100 \mathrm{mM}$ glycine and $2 \%$ bovine serum albumin (BSA; Sigma-Aldrich; Merck KGaA, Darmstadt, Germany) in $1 \mathrm{X}$ PBS (PBSB). Beads enriched with EVs were washed once using PBSB for $5 \mathrm{~min}$ then blocked with $10 \%$ BSA for $30 \mathrm{~min}$ at room temperature. Following two washes with PBSB for $5 \mathrm{~min}$, the beads were incubated with GPC1 antibody (1:6; GTX 104557; GeneTex, Inc., Irvine, CA, USA) on ice for $1 \mathrm{~h}$ and washed twice with PBSB for 5 min. Subsequently, the EV-bound beads were incubated with an Alexa-488-conjugated anti-rabbit secondary antibody (1:2,000; ab150077; Abcam, Cambridge, UK) for $30 \mathrm{~min}$ at $4^{\circ} \mathrm{C}$. EV-bound beads that did not undergo primary antibody incubation were used as a negative control. The events were collected with a flow cytometer and then the single beads and the doublets of beads without EVs were gated according to the Forward Scatter and Side Scatter. EV-coated beads, with or without reaction with GPC1 antibodies, were collected, and the fluorescence intensity was analyzed. The percentage of $\mathrm{GPC}^{+}$ 
EVs was calculated as the number of $\mathrm{GPC1}^{+} \mathrm{EVs}$ relative to the total number of beads per sample (100,000 events). This percentage was therein referred to as the percent beads with $\mathrm{GPC}^{+}$EVs. Similarly, EV surface markers were analyzed using fluorescein isothiocyanate-conjugated CD63 and CD81 antibodies at $4^{\circ} \mathrm{C}$ for $30 \mathrm{~min}(1: 500 ; 557288$ and 551108 ; BD Biosciences, San Jose, CA, USA). All flow cytometry analysis was conducted using a BD accuri C6 flow cytometer system (BD Biosciences). Results were analyzed with FlowJo software version 8.7.3 (Tree Star Inc., Ashland, OR, USA).

Statistical analysis. Data are presented as mean \pm standard deviation, median (25 and 75th quartiles) or mean (range). An unpaired Student's t-test was applied to calculate the statistical significance between two independent groups. Wilcoxon signed rank test was performed to compare the difference in the proportion of $\mathrm{GPCl}^{+} \mathrm{EVs}$ between blood samples collected pre- and post-RIAC treatment. The survival curves for overall survival were analyzed using the Kaplan-Meier method and compared using a log-rank test in the longitudinal cohort of patients with APC. All statistical analyses were performed using SPSS version 16.0 (SPSS, Inc., Chicago, IL, USA). $\mathrm{P}<0.05$ was considered to indicate a statistically significant difference.

\section{Results}

Patient characteristics. A total of 28 patients with APC were enrolled in the present study. All patients received two cycles of RIAC. As presented in Table I, the mean patient age was 65 years (range, 43-83 years). Of these, $15(53.5 \%)$ patients were diagnosed with lesions in the head of the pancreas. The reference values of CA19-9 and CEA in healthy population were $10-37 \mathrm{U} / \mathrm{ml}$ and $0-5 \mathrm{ng} / \mathrm{ml}$, respectively (23). In the present study, the median values of CA19-9 and CEA in patients were $851.4(14.5,3,765.0) \mathrm{U} / \mathrm{ml}$ and $5.3(2.6,14.6) \mathrm{ng} / \mathrm{ml}$, respectively. The median overall survival time was 6.0 months $(95 \%$ confidence interval, 4.9-7.9 months).

$\mathrm{GPCl}^{+} \mathrm{EVs}$ in patients with advanced pancreatic cancer. EVs were obtained from plasma using a spin column-based method. Transmission electron microscopy was used to visually inspect the size and morphology of EVs. As hypothesized, the size of EVs ranged between 50 and $200 \mathrm{~nm}$ (Fig. 1A and B). Furthermore, specific exosome markers (CD63 and CD81) were examined using flow cytometry. However, EVs only partially expressed CD63 (59.2\%) and CD81 (74.1\%) (Fig. 1C and D). These data indicated that EVs isolated from plasma using this method were composed of other EVs, besides exosomes. Next, $\mathrm{GPC1}^{+} \mathrm{EVs}$ were examined in patients with APC $(n=28)$ and age- and gender-matched healthy individuals $(n=16)$ by flow cytometry analysis. As presented in Fig. 2, the levels of $\mathrm{GPCl}^{+} \mathrm{EVs}$ in patients with APC (39.8 $\left.\pm 19.0 \%\right)$ were significantly increased compared with corresponding controls $(1.02 \pm 0.62 \% ; \mathrm{P}<0.001)$.

$\mathrm{GPCl}^{+}$EVs in patients with pancreatic cancer following RIAC treatment. RIAC is an alternative treatment for patients with APC who are not suitable for traditional surgery. Therefore,
Table I. Demographic and clinical characteristics of patients.

\begin{tabular}{lc}
\hline Variables & Value \\
\hline Gender & \\
Male, $\mathrm{n}(\%)$ & $16(57.1)$ \\
Female, $\mathrm{n}(\%)$ & $12(42.9)$ \\
Age, years ${ }^{\mathrm{a}}$ & $65(43-83)$ \\
Tumor location & \\
Head, n $(\%)$ & $15(53.6)$ \\
Body-Tail, n $(\%)$ & $13(46.4)$ \\
CA19-9, U/ml ${ }^{\mathrm{b}}$ & $851.4(14.5-3,765.0)$ \\
CEA, ng/ml ${ }^{\mathrm{b}}$ & $5.3(2.6-14.6)$ \\
TNM stage & \\
I, n $(\%)$ & $4(14.3)$ \\
II, n $(\%)$ & $9(32.1)$ \\
III, n $(\%)$ & $15(53.6)$ \\
\hline
\end{tabular}

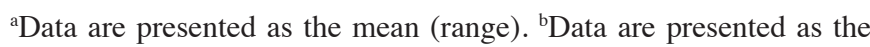
median (interquartile range). CA19-9, cancer antigen 19-9; CEA, carcinoembryonic antigen; TNM, Tumor-Node-Metastasis.

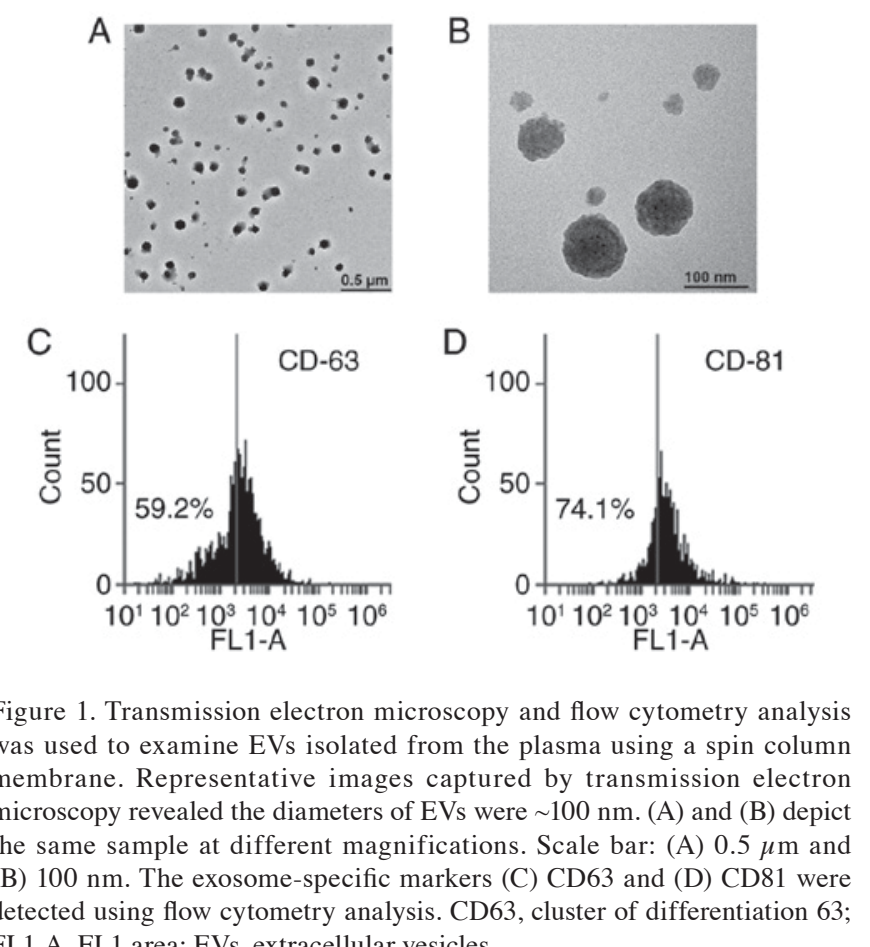
FL1-A, FL1 area; EVs, extracellular vesicles.

we hypothesized that $\mathrm{GPC1}^{+} \mathrm{EVs}$ may be suitable as a clinical marker to predict the outcome of RIAC treatment for patients with APC. Levels of $\mathrm{GPC}^{+} \mathrm{EV}^{+}$were measured in the plasma of patients with APC $(n=28)$ following RIAC treatment. The results of the present study revealed that the mean proportion of $\mathrm{GPC}^{+}$EVs was $37.1 \%$ (range, 11-83\%) following RIAC treatment. Compared with pre-RIAC treatment, levels of $\mathrm{GPC}^{+}$ EVs were significantly reduced in patients with $\mathrm{APC}(\mathrm{P}=0.023)$ (Fig. 3). These data indicated that $\mathrm{GPC1}^{+} \mathrm{EVs}_{\mathrm{s}}$ possess the potential to be a prognostic maker for patients with APC. 


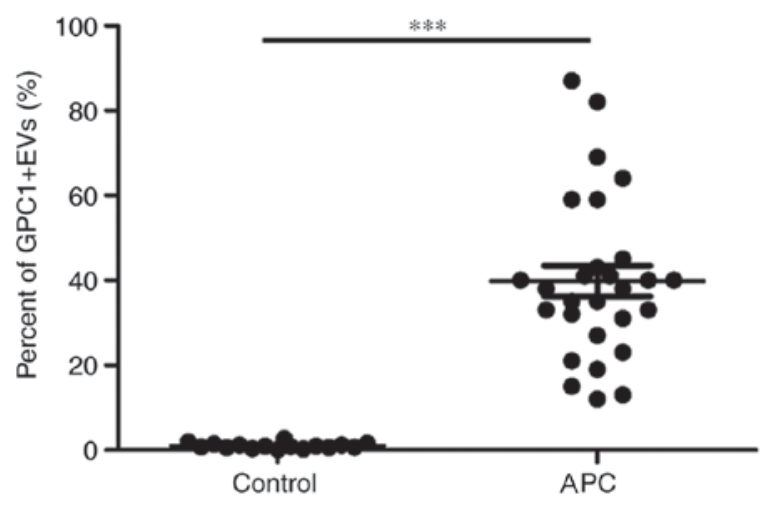

Figure 2. Percentage of $\mathrm{GPCl}^{+} \mathrm{EVs}$ were increased in patients with APC. The figure depicts the percentage of beads with $\mathrm{GPCl}^{+} \mathrm{EVs}$ in healthy controls $(\mathrm{n}=16)$ and patients with APC $(\mathrm{n}=28) .{ }^{* * * *} \mathrm{P}<0.001$. EVs, extracellular vesicles; $\mathrm{GPCl}^{+}$, glypican-1; APC, advanced pancreatic cancer.

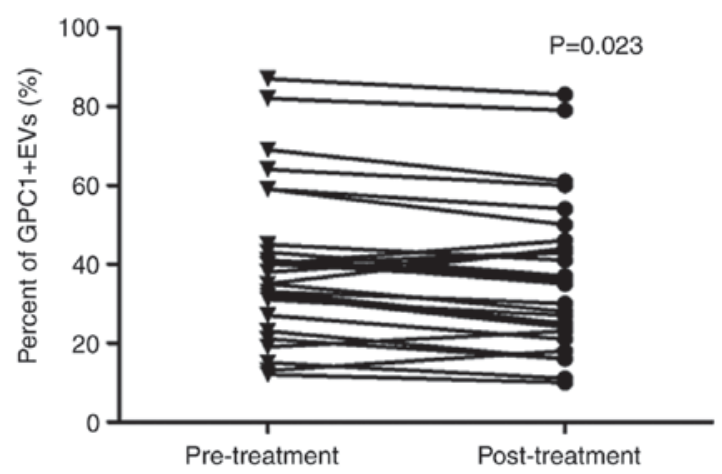

Figure 3. $\mathrm{GPCl}^{+} \mathrm{EVs}$ levels were reduced following RIAC treatment. Changes in $\mathrm{GPCl}^{+} \mathrm{EVs}$ levels in 28 patients with APC prior to and following RIAC treatment. EVs, extracellular vesicles; $\mathrm{GPCl}^{+}$, glypican-1; APC, advanced pancreatic cancer; RIAC, regional intra-arterial chemotherapy.

Longitudinal evaluation of plasma $G P C 1^{+}$EVs. The prognostic relevance of $\mathrm{GPCl}^{+} \mathrm{EVs}$ and overall survival rates, assessed using Kaplan-Meier curves, were determined in the present longitudinal study. Patients were divided into two groups, higher or lower than the median $\mathrm{GPC}^{+} \mathrm{EV}$ level. There was a tendency towards reduced overall survival rates for patients with high levels of $\mathrm{GPCl}^{+} \mathrm{EVs}$ detected prior to RIAC treatment ( $\mathrm{P}=0.151)$ (Fig. 4A). Furthermore, a reduced overall survival rate was associated with increased levels of $\mathrm{GPCl}^{+}$EVs following RIAC treatment $(\mathrm{P}=0.267)$ (Fig. 4B). A greater decrease in the proportion of $\mathrm{GPCl}^{+} \mathrm{EVs}$ following RIAC treatment was associated with an improved median overall survival rate compared with lesser decreases in $\mathrm{GPCl}^{+}$ EVs ( $\mathrm{P}=0.03)$ (Fig. 4C). These data provide evidence that the decreased level of $\mathrm{GPC}^{+} \mathrm{EVs}$ could be utilized as prognostic marker to predict the outcome of RIAC treatment in patients with APC.

\section{Discussion}

Pancreatic cancer is a highly lethal malignancy, owing to its aggressive nature and the lack of early detection modalities $(24,25)$. RIAC is a potential alternative treatment for APC and may be useful to prolong survival of patients $(5,8,18,19,26)$. EVs carry proteins, lipids, and RNAs, mediating communication between different cell types in the body, and thus affect normal and pathological conditions (27). A previous study has identified that $\mathrm{GPCl}^{+} \mathrm{EVs}$ may be detected in patients with pancreatic cancer at early stages of the disease (13). However, it remains unknown whether GPC1 could be used to predict the response of RIAC for patients with APC. The present study primarily focused on the research concerning arterial infusion in cancer treatment. Therefore, an attempt was made to assess whether the proportion of $\mathrm{GPCl}^{+} \mathrm{EVs}$ could serve as a novel predictor of RIAC outcome for patients with APC. In the present study, EVs were isolated from the plasma of patients with APC and it was identified that levels of $\mathrm{GPC}^{+} \mathrm{EVs}$ were reduced following RIAC therapy. It was subsequently identified that a greater decrease of $\mathrm{GPC}^{+}$EVs following RIAC treatment was associated with improved survival rates of patients with APC. The present study provides novel insights that $\mathrm{GPC}^{+}$ EVs may therefore represent useful prognostic biomarkers for evaluating the outcome of RIAC treatment in patients with APC.

Pancreatic cancer is a disease with a low overall survival rate; the incidence rate of this disease is expected to continue increasing (7). Surgical resection and systemic chemotherapy are common treatment modalities for patients with pancreatic cancer $(28,29)$. Surgical resection is the only potentially curative treatment for pancreatic cancer; however, $85 \%$ of patients have progressed to an advanced unresectable stage at the time of diagnosis (3). For patients who are not suitable candidates for surgery, systemic monotherapy with gemcitabine or 5-fluorouracil is widely considered the standard treatment for pancreatic cancer. However, this treatment has a low response rate and increases the risk of severe side effects, including cytopenia and bone marrow suppression $(30,31)$. Arterial infusion, including RIAC, has been used as an alternative strategy to increase the survival rates for patients with pancreatic cancer $(19,30,32)$. RIAC delivers a high concentration of drugs into the artery connected to the tumor and provides patients an optimal therapeutic approach, particularly for APC $(33,34)$. Although RIAC is not a routine treatment for pancreatic cancer, clinical trials have identified that RIAC achieves a significant benefit in terms of overall survival rates and symptomatic control in patients with pancreatic cancer $(5,32)$.

Previously, pain alleviation and changes in body weight were used to assess the clinical benefit of treatment. CA19-9 and CEA levels are commonly used to determine the prognosis of patients with APC; however, their sensitivities and specificities may be insufficient to assess treatment benefit $(35,36)$. As a consequence, the results were controversial when evaluating the safety and efficacy of treatments, including systemic chemotherapy and surgery $(37,38)$. Therefore, identification of a reliable biomarker to evaluate the effect of RIAC in APC treatment remains a challenge for researchers. Extensive research on EVs is being conducted, since they represent a source of non-invasive biomarkers to accurately predict disease prognosis in numerous types of cancer $(12,39)$. Evidence indicates that GPC1 is overexpressed in patients with pancreatic adenocarcinoma, which directly contributes to the carcinogenesis and development 

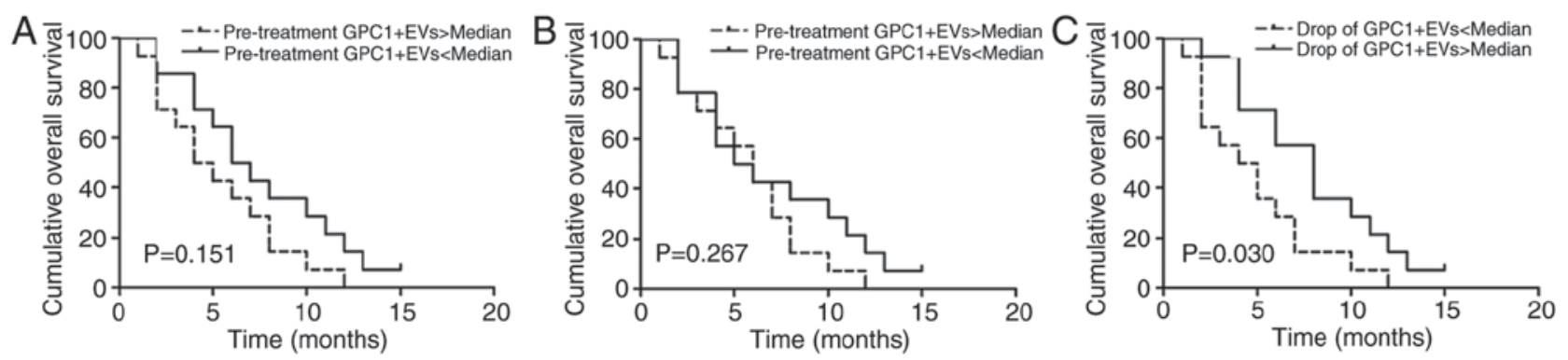

Figure 4. Kaplan-Meier curves for overall survival rates in patients with a greater or lesser proportion of GPC1 ${ }^{+}$EVs than the median (A) prior to RIAC treatment, (B) following RIAC treatment, and (C) in those that exhibited a fall in the proportion of $\mathrm{GPC1}^{+}$EVs. EVs, extracellular vesicles; GPC1 ${ }^{+}$, glypican-1; APC, advanced pancreatic cancer; RIAC, regional intra-arterial chemotherapy.

of pancreatic adenocarcinoma by controlling cell proliferation (15,17). Previously, Melo et al (13) demonstrated that $\mathrm{GPC}^{+}$exosomes could be detected in the serum of patients with pancreatic cancer at early stages of disease. Consistent with this previous report, the present study also confirmed that $\mathrm{GPC}^{+} \mathrm{EVs}$ were highly expressed in patients with APC. Thus, the present study further confirmed the utility of $\mathrm{GPCl}^{+} \mathrm{EVs}$ as a potential biomarker for APC diagnosis. Furthermore, it was revealed that a greater decrease of $\mathrm{GPCl}^{+}$EVs following RIAC treatment was associated with improved survival rates of patients with APC, indicating that $\mathrm{GPC1}^{+} \mathrm{EVs}$ could also be used as a prognostic marker for patients with APC receiving RIAC treatment. Although considerable attention was generated by the applications of EVs in disease diagnostics and prognosis, the methods of purifying EVs from plasma remain non-optimal $(40,41)$. In the present study, a spin column-based method was used to isolate EVs from plasma (22). This approach represented an improvement on traditional methods such as differential centrifugation, and is a more convenient method to obtain a high quantity of EVs from plasma.

In the present study, plasma EVs were isolated from the blood of patients with APC, and it was identified that levels of $\mathrm{GPCl}^{+} \mathrm{EVs}$ were increased in patients with APC and were decreased following RIAC treatment. Furthermore, it was identified that a greater decrease in $\mathrm{GPCl}^{+} \mathrm{EVs}$ was associated with increased survival rates. The present study demonstrated that $\mathrm{GPCl}^{+} \mathrm{EVs}$ could be utilized to evaluate the outcome of RIAC treatment for patients with APC. In the present study, only 28 patients with APC were enrolled since strict criteria were adopted to minimize potential confounding effects. However, the statistical estimation calculated prior to the initiation of the study identified that only 18 patients were required when the threshold of mean difference between preand post-RIAC treatment was taken to be $5 \%$ and the expected power was set at $50 \%$, using a significance level $\alpha=0.05$. To validate the prognostic value of $\mathrm{GPC}^{+} \mathrm{EVs}$ for assessing the efficacy of RIAC treatment in patients with APC, future studies are required to increase the sample size with a large independent cohort.

In conclusion, the present study revealed that the proportion of $\mathrm{GPCl}^{+} \mathrm{EVs}$ was higher in patients with APC, and that this decreased following RIAC treatment. Furthermore, it was revealed that a greater decrease of $\mathrm{GPC}^{+} \mathrm{EVs}$ following RIAC treatment was associated with improved survival rates of patients with APC. These data provide a novel insight into the central functions of EVs in the diagnosis of cancer.

\section{Acknowledgements}

The authors would like to thank Dr Ke-Feng $\mathrm{Pu}$ (Soochow University, Suzhou, China) for technical assistance in performance of the transmission electron microscopy.

\section{Funding}

The study was supported by the Natural Science Foundation of China (grant no, 81770483), Jiangsu Provincial Science and Technology office (grant no. BL2014043), and Suzhou Health and Family Planning Commission Program (grant no. LCZX201504).

\section{Availability of data and materials}

The datasets used and/or analyzed during the current study are available from the corresponding author on reasonable request.

\section{Authors' contributions}

JYQ and XQL conceived and coordinated the study and wrote the paper. YLT, YZ, YFY and JYQ performed and analyzed the experiments. JYQ contributed to the preparation of sample.

\section{Ethics approval and consent to participate}

Written informed consent was obtained from all study participants prior to participating in the study. The study was approved by the Institutional Research Ethic Board at The Bengbu Medical College.

\section{Consent for publication}

Written informed consent was obtained from all study participants for publication of the study.

\section{Competing interests}

The authors declare that they have no competing interests. 


\section{References}

1. Ryan DP, Hong TS and Bardeesy N: Pancreatic adenocarcinoma. New Engl J Med 371: 1039-1049, 2014.

2. Siegel RL, Miller KD and Jemal A: Cancer statistics, 2015. CA Cancer J Clin 65: 5-29, 2015.

3. Paniccia A, Hosokawa P, Henderson W, Schulick RD, Edil BH, McCarter MD and Gajdos C: Characteristics of 10-year survivors of pancreatic ductal adenocarcinoma. JAMA Surg 150: 701-710, 2015.

4. Conlon KC, Klimstra DS and Brennan MF: Long-term survival after curative resection for pancreatic ductal adenocarcinoma Clinicopathologic analysis of 5-year survivors. Ann Surg 223 273-279, 1996

5. Jin C, Yao L, Long J, Fu DL, Yu XJ, Xu J, Yang F and Ni QX: Effect of multiple-phase regional intra-arterial infusion chemotherapy on patients with resectable pancreatic head adenocarcinoma. Chinese Med J (Engl) 122: 284-290, 2009.

6. Sasada T, Denno R, Tanaka T, Kanai M, Mizukami Y, Kohno S and Takabayashi A: Intra-arterial infusion chemotherapy with 5-fluorouracil and cisplatin in advanced pancreatic cancer: A feasibility study. Am J Clin Oncol 31: 71-78, 2008.

7. Shi S, Yao WT, Xu J, Long J, Liu C and Yu XJ: Combinational therapy: New hope for pancreatic cancer? Cancer Lett 317: $127-135,2012$

8. Ohigashi H, Ishikawa O, Imaoka S, Sasaki Y, Kabuto T, Kameyama M, Furukawa H, Hiratuka M, Nakamori S, Nakano H, et al: A new method of intra-arterial regional chemotherapy with more selective drug delivery for locally advanced pancreatic cancer. Hepatogastroenterology 43: 338-345, 1996.

9. Swords DS, Firpo MA, Scaife CL and Mulvihill SJ: Biomarkers in pancreatic adenocarcinoma: Current perspectives. Onco Targets Ther 9: 7459-7467, 2016.

10. Raposo G and Stoorvogel W: Extracellular vesicles: Exosomes, microvesicles, and friends. J Cell Biol 200: 373-383, 2013.

11. van der Pol E, Böing AN, Harrison P, Sturk A and Nieuwland R: Classification, functions, and clinical relevance of extracellular vesicles. Pharmacol Rev 64: 676-705, 2012.

12. Sharma A, Khatun Z and Shiras A: Tumor exosomes: Cellular postmen of cancer diagnosis and personalized therapy. Nanomedicine (Lond) 11: 421-437, 2016.

13. Melo SA, Luecke LB, Kahlert C, Fernandez AF, Gammon ST, Kaye J, LeBleu VS, Mittendorf EA, Weitz J, Rahbari N, et al: Glypican-1 identifies cancer exosomes and detects early pancreatic cancer. Nature 523: 177-182, 2015.

14. Kayed H, Kleeff J, Keleg S, Jiang XH, Penzel R, Giese T, Zentgraf H, Büchler MW, Korc M and Friess H: Correlation of glypican-1 expression with TGF-beta, BMP, and activin receptors in pancreatic ductal adenocarcinoma. Int J Oncol 29: 1139-1148, 2006.

15. Duan L, Hu XQ, Feng DY, Lei SY and Hu GH: GPC-1 may serve as a predictor of perineural invasion and a prognosticator of survival in pancreatic cancer. Asian J Surg 36: 7-12, 2013.

16. Li C, Du X, Tai S, Zhong X, Wang Z, Hu Z, Zhang L, Kang P, Ji D, Jiang X, et al: GPC1 regulated by miR-96-5p, rather than miR-182-5p, in inhibition of pancreatic carcinoma cell proliferation. Int J Mol Sci 15: 6314-6327, 2014.

17. Kleeff J, Ishiwata T, Kumbasar A, Friess H, Büchler MW, Lander AD and Korc M: The cell-surface heparan sulfate proteoglycan glypican-1 regulates growth factor action in pancreatic carcinoma cells and is overexpressed in human pancreatic cancer. J Clin Invest 102: 1662-1673, 1998.

18. Liu FH, Tang Y, Sun J, Yuan Z, Li S, Sheng J, Ren H and Hao J: Regional intra-arterial vs. systemic chemotherapy for advanced pancreatic cancer: A systematic review and meta-analysis of randomized controlled trials. PloS One 7 : e40847, 2012.

19. Liu X, Yang X, Zhou G, Chen Y, Li C and Wang X: Gemcitabine-based regional intra-arterial infusion chemotherapy in patients with advanced pancreatic adenocarcinoma. Medicine (Baltimore) 95: e3098, 2016.

20. Corke $C$ and Cranswick P: A Seldinger technique for minitracheostomy insertion. Anaesth Intensive Care 16: 206-207, 1988.

21. Katz MH, Hwang R, Fleming JB and Evans DB: Tumor-node-metastasis staging of pancreatic adenocarcinoma CA Cancer J Clin 58: 111-125, 2008.
22. Enderle D, Spiel A, Coticchia CM, Berghoff E, Mueller R, Schlumpberger M, Sprenger-Haussels M, Shaffer JM, Lader E, Skog J and Noerholm M: Characterization of RNA from exosomes and other extracellular vesicles isolated by a novel spin column-based method. PloS One 10: e0136133, 2015.

23. Nazli O, Bozdag AD, Tansug T, Kir R and Kaymak E: The diagnostic importance of CEA and CA 19-9 for the early diagnosis of pancreatic carcinoma. Hepatogastroenterology 47: 1750-1752, 2000.

24. Kamisawa T, Wood LD, Itoi T and Takaori K: Pancreatic cancer. Lancet 388: 73-85, 2016.

25. Mohammed S, Van Buren G II and Fisher WE: Pancreatic cancer: Advances in treatment. World J Gastroenterol 20: 9354-9360, 2014.

26. Davis JL, Pandalai P, Ripley RT, Langan RC, Steinberg SM, Walker M, Toomey MA, Levy E and Avital I: Regional chemotherapy in locally advanced pancreatic cancer: RECLAP trial. Trials 12: 129, 2011.

27. Colombo M, Raposo G and Théry C: Biogenesis, secretion, and intercellular interactions of exosomes and other extracellular vesicles. Annu Rev Cell Dev Biol 30: 255-289, 2014.

28. Muñoz Martín AJ, Adeva J, Martínez-Galán J, Reina JJ and Hidalgo M: Pancreatic ductal adenocarcinoma: Metastatic disease. Clin Transl Oncol 19: 1423-1429, 2017.

29. Conroy T, Bachet JB, Ayav A, Huguet F, Lambert A, Caramella C, Maréchal R, Van Laethem JL and Ducreux M: Current standards and new innovative approaches for treatment of pancreatic cancer. Eur J Cancer 57: 10-22, 2016.

30. Cardenes HR, Chiorean EG, Dewitt J, Schmidt M and Loehrer P: Locally advanced pancreatic cancer: Current therapeutic approach. Oncologist 11: 612-623, 2006.

31. Burris HA III, Moore MJ, Andersen J, Green MR, Rothenberg ML, Madiano MR, Cripps MC, Portenoy RK, Storniolo AM, Tarassoff P, et al: Improvements in survival and clinical benefit with gemcitabine as first-line therapy for patients with advanced pancreas cancer: A randomized trial. J Clin Oncol 15: 2403-2413, 1997.

32. Wang Z, Ren ZG, Ma NY, Zhao JD, Zhang Z, Ma XJ, Long J, $\mathrm{Xu}$ J and Jiang GL: Intensity modulated radiotherapy for locally advanced and metastatic pancreatic cancer: A mono-institutional retrospective analysis. Radiat Oncol 10: 14, 2015.

33. Takamori H, Kanemitsu K, Tsuji T, Tanaka H, Chikamoto A, Nakahara O, Hiraoka T, Ikeda O, Kudo K, Imuta M and Yamashita Y: 5-fluorouracil intra-arterial infusion combined with systemic gemcitabine for unresectable pancreatic cancer. Pancreas 30: 223-226, 2005.

34. Muchmore JH, Preslan JE and George WJ: Regional chemotherapy for inoperable pancreatic carcinoma. Cancer 78 (3 Suppl): S664-S673, 1996.

35. Torres C, Perales S, Alejandre MJ, Iglesias J, Palomino RJ, Martin M, Caba O, Prados JC, Aránega A, Delgado JR, et al: Serum cytokine profile in patients with pancreatic cancer. Pancreas 43: 1042-1049, 2014.

36. Zhang Y, Yang J, Li H, Wu Y, Zhang H and Chen W: Tumor markers CA19-9, CA242 and CEA in the diagnosis of pancreatic cancer: A meta-analysis. Int J Clin Exp Med 8: 11683-11691, 2015.

37. Grønborg M, Kristiansen TZ, Iwahori A, Chang R, Reddy R, Sato N, Molina H, Jensen ON, Hruban RH, Goggins MG, et al: Biomarker discovery from pancreatic cancer secretome using a differential proteomic approach. Mol Cell Proteomics 5: 157-171, 2006.

38. Zhang L, Farrell JJ, Zhou H, Elashoff D, Akin D, Park NH, Chia D and Wong DT: Salivary transcriptomic biomarkers for detection of resectable pancreatic cancer. Gastroenterology 138: 949-957.e1-e7, 2010

39. Kalluri R: The biology and function of exosomes in cancer. J Clin Invest 126: 1208-1215, 2016.

40. Muller L, Hong CS, Stolz DB, Watkins SC and Whiteside TL: Isolation of biologically-active exosomes from human plasma. J Immunol Methods 411: 55-65, 2014.

41. Kalra H, Adda CG, Liem M, Ang CS, Mechler A, Simpson RJ, Hulett MD and Mathivanan S: Comparative proteomics evaluation of plasma exosome isolation techniques and assessment of the stability of exosomes in normal human blood plasma. Proteomics 13: 3354-3364, 2013. 